\title{
Comunicación Preliminar Acerca de la Disposición Morfológica, la Funcionalidad y las Implicancias Clínicas del Modiolo del Ángulo Oral (Modiolus anguli oris)
}

\author{
Preliminary Communication About the Morphological Arrangement, Functionality \\ and Clinical Implications of Modiolus of Angulus Oris (Modiolus anguli oris)
}

\author{
Nicolás E. Ottone $e^{1,2,3}$
}

OTTONE, N. E. Comunicación preliminar acerca de la disposición morfológica, la funcionalidad y las implicancias clínicas del modiolo del ángulo oral (Modiolus anguli oris). Int. J. Morphol. 38(6):1837-1841, 2020.

RESUMEN: La disposición anatómica de los músculos faciales es de una notable complejidad y requiere de un estudio pormenorizado para lograr su reconomiento preciso. La comprensión de la disposición morfológica y la funcionalidad del modiolo del ángulo oral (Modiolus angulis oris) es de gran relevancia al momento de analizar la constitución muscular de la comisura labial, y para comprender clínicamente las lesiones del ángulo de la cavidad oral, lo que permitirá un abordaje terapéutico adecuado, crucial para la preservación y reconstitución de la armonía facial.

PALABRAS CLAVE: Modiolo del ángulo oral; Modiolus angulis oris; Morfología; Anatomía clínica.

\section{INTRODUCCIÓN}

La disección de los músculos faciales, en comparación a otra región anatómica (sólo quizás comparable a la región perineal), es en extremo compleja, y requiere ser llevada adelante por un disector experimentado, ya que la disposición muscular, vascular y especialmente nerviosa de la región, necesita de gran precisión y tiempo para el descubrimiento a salvo de todos los elementos anatómicos. Estos músculos, por lo general, son tratados muy superficialmente en la mayoría de los libros de anatomía (D'Andrea \& Barbaix, 2006). Sin embargo, sutiles y muy diversas expresiones faciales se logran por la actividad e interacción simultánea de los músculos de la mímica (Yu et al., 2013), participando en diferentes funciones como masticar, tragar, fonación y expresiones mímicas (D'Andrea \& Barbaix).

En este sentido, la comisura labial constituye un punto de reunión y entrecruzamiento de diversos músculos faciales, los cuales van a constituir el modiolo del ángulo oral (Modiolus angulis oris), estructura funcional y estética fundamental de la porción inferior de la cara, constituyéndose como una unidad estética natural (Kim et al., 2015). Es importante enton- ces comprender de mejor manera la anatomía de esta región al intentar el rejuvenecimiento facial (Ewart et al., 2005).

El modiolo del ángulo oral se encuentra lateral y ligeramente superior a cada ángulo oral (Hotta, 2016). El modiolo del ángulo oral es un área de convergencia de fibras musculares que forma un nódulo tendinoso que se adhiere firmemente a la dermis en el ángulo oral. Los músculos que se insertan en el modiolo del ángulo oral tienen el mayor impacto en la apariencia de las comisuras labiales (Ewart et al.).

El modiolo del ángulo oral tiene un alto grado de complejidad tridimensional, con algunos de los músculos orientados en el plano oblicuo de la cara (Sun et al., 2018). Estos músculos comúnmente se superponen entre sí, lo que los hace difíciles de seguir.

El objetivo de este trabajo consistió en caracterizar la morfología del modiolo del ángulo oral, identificando su funcionalidad y las implicancias clínicas para un correcto abordaje terapéutico de la región perioral.

\footnotetext{
${ }^{1}$ Laboratorio de Plastinación y Técnicas Anatómicas, Facultad de Odontología, Universidad de La Frontera, Temuco, Chile.

${ }^{2}$ Centro de Excelencia en Estudios Morfológicos y Quirúrgicos (CEMyQ), Universidad de La Frontera, Temuco, Chile.

${ }^{3}$ Programa de Doctorado en Ciencias Morfológicas, Universidad de La Frontera, Temuco, Chile.

Financiado por la Dirección de Investigación y Postgrado de la Universidad de La Frontera, a través del Proyecto de Investigación Asociativa Investigadores Pertenecientes a las Facultades IAF 2018, Proyecto No AF18-0014.
} 


\section{MATERIAL Y MÉTODO}

Búsqueda científica. Se realizó una búsqueda de la literatura en las bases de datos Pubmed, Scopus y SciELO, con las siguientes estrategias de búsqueda: en Pubmed y Scopus, (modiolus) AND (anatomy) AND (human) AND (oral); en Scielo, (modiolo) AND (anatomia) AND (humanos) AND (oral). En relación a los criterios de inclusión, se consideraron todos los artículos que tuvieran como temática principal el estudio del modiolo y/o los músculos constituyentes en forma particular, tanto con orientación puramente anatómica como así también clínica. Asimismo, se consideraron los artículos en español e inglés, que trataran trabajos en humanos. No hubo exclusión por tiempo de publicación. La búsqueda en las tres bases de datos permitió encontrar un total de 39 artículos (Pubmed, 28 artículos; Scopus, 10 artículos; SciELO, 1 artículo). Del total de artículos, se eliminaron los duplicados, quedando 33 artículos. Finalmente, los 33 artículos fueron revisados siguiendo los criterios de inclusión y exclusión considerados, seleccionándose 14 artículos. Se incorporaron dos libros a la literatura consultada, quedando un total de 16 referencias bibliográficas para el presente trabajo.

Técnica de disección de la musculatura perioral desarrollada por el Dr. José Donaldo Giacomotti (Giacomotti et al., 2009). De la literatura consultada, la mayoría realizaba el abordaje y disección de la musculatura perioral de una manera tradicioal de superficial (piel) a profundo. Sin embargo, es necesario resaltar la metodología desarrollada por el Dr. José Donaldo Giacomotti, destacado y reconocido Médico, Cirujano Plástico y Profesor de Anatomía de la Facultad de Medicina de la Univesidad de Buenos Aires, Argentina. Fue el creador y es actual director del Museo de Anatomía de la Segunda Cátedra de Anatomía de la Universidad de Buenos Aires, a partir de cuyas enseñanzas y dirección gran cantidad de estudiantes de medicina colaboraron con la disección de más de 400 preparaciones anatómicas expuestas en el museo. En este contexto, es importante destacar la particular técnica de disección desarrollada para el abordaje e identificación de los músculos faciales. Para ello, el Dr. Giacomotti iniciaba la disección desde el plano del periostio, buscando alcanzar y abordar la musculatura por su cara profunda, permitiendo de esta manera individualizar progresivamente cada uno de los músculos periorales. En este sentido, se debe extraer en bloque toda la región muscular perioral desde el periostio y este bloque muscular es el que se aborda al momento de la disección. En un segundo momento, se afronta la disección desde la superficie, abordando la región desde los planos cutáneo y celular subcutáneo, hasta alcanzar la cara superficial de la musculatura. De esta manera, el abordaje de la región para su disección es doble (por superficial y profundo) con lo que se lo- gra identificar con gran precisión, y de una manera global, cada una de las estructuras anatómicas constituyentes del sistema muscular perioral, pudiendo indentificar claramente el recorrido preciso de los distintos fascículos musculares (Giacomotti et al.).

\section{RESULTADOS Y DISCUSIÓN}

La zona paracomisural (Giacomotti et al.) es considerada una zona de entrecruzamiento de fibras musculares (Lightoller, 1925; Freilinger et al., 1987; Williams, 1988; D’Andrea \& Barbaix; Shim et al., 2008; Giacomotti et al.; Yu et al.; Kim et al.; Hur, 2018; Tong et al., 2019), las cuales determinan la formación de un núcleo muscular fibroso denominado modiolo del ángulo oral (Modiolus anguli oris). El modiolo del ángulo oral se podría definir como el origen o terminación de los músculos levantador del ángulo oral (Levator anguli oris), depresor del ángulo oral y músculo cigomático mayor, lo cual justificaría el funcionamiento de cada uno.

A su vez, el modiolo del ángulo oral está asociado a diversas teorías en relación a la formación del músculo orbicular oral. Según varios autores (Rubin, 1974; D’Andrea \& Barbaix; Giacomotti et al.) este músculo está formado por fascículos emanados de los músculos periorales que confluyen y se entrecruzan en la zona paracomisural, perdiendo su supuesta unidad individual, y colaborando también en la formación del modiolo del ángulo oral (Modiolus anguli oris), a partir del cual también se enviarían las fibras musculares hacia los labios. Por su parte, Kim et al. consideran al modiolo del ángulo oral como una estructura muscular que se decusa entre el músculo orbicular oral y los músculos que terminan en el margen lateral del vértice de la cavidad oral. De esta manera considera como una unidad individual al músculo orbicular oral, a diferencia de lo identificado por Rubin, D'Andrea \& Barbaix y Giacomotti et al., considerando además que el levantador del labio superior pasa a través del músculo orbicular oral y participa en la formación del pliegue nasolabial (Kim et al.). Kim et al. también definieron al modiolo del ángulo oral como un pequeño nódulo en forma de botón de tejido conectivo que se entrelaza con ciertos músculos faciales en cada lado.

Al-Hoqail \& Abdel Meguid (2009) señalaron que las estructuras anatómicas que componen el modiolo del ángulo oral corresponden a las fascias superficial y profunda de la cara, como así también a los siguientes músculos: cigomático mayor, buccinador, platisma porción modiolar, porción marginal del orbicular, levantador del ángulo oral, mental, depresor del labio inferior, depresor del ángulo oral 
y risorio. Para Choi et al. (2014) el modiolo del ángulo oral es una masa muscular móvil ubicada en el margen lateral de la comisura labial, y estaba formada por la convergencia de las fibras musculares de los músculos depresor del ángulo oral, cigomático mayor, orbicular oral, risorio, buccinador y levantador del ángulo oral.

Shim et al. estudiaron el músculo cigomático mayor, identificando su inserción en el modiolo del ángulo oral, y el entrelazamiento de sus fibras musculares con las de los músculos buccinador, levantador del ángulo oral y orbicular oral. Al-Hoqail \& Abdel Meguid identificaron las fascias profunda y superficial de la cara en relación al modiolo del ángulo oral, concluyendo que las mismas no convergen por delante del músculo masetero sino en el modiolo del ángulo oral. Esto fue confirmado por Kim et al. quienes encontraron que la fascia superficial de la cara continúa medial al surco nasolabial y converge en el modiolo del ángulo oral. La fascia, que es un tejido conectivo no contráctil, puede actuar para estabilizar el músculo, los nervios y los vasos. Por su parte, Yu et al. también encontraron a las fascias superficial y profunda de la cara convergiendo en el modiolo del ángulo oral; en particular, la fascia superficial se atenuaba medialmente en el modiolo del ángulo oral, desapareciendo y mezclándose con la musculatura facial responsable del movimiento y los labios.

Al-Hoqail \& Abdel Meguid identificaron también diversas disposiciones de la arteria facial con el modiolo del ángulo oral, encontrándola anterior al modiolo del ángulo oral en el $20 \%$ de los casos estudiados, y lateral al modiolo del ángulo oral en el $80 \%$ restante. Nunca encontraron la arteria facial medial al modiolo del ángulo oral, como así tampoco un canal fibroso o una hendidura en el ángulo oral a nivel del modiolo del ángulo oral. Por su parte, Sullivan et al. (2010) identificaron que la arteria facial vasculariza al modiolo del ángulo oral en la región perioral y transcurre a lo largo de su porción lateral para terminar como arteria angular.

Shim et al. consideran crucial la relación existente entre la banda muscular profunda del músculo cigomático mayor y el músculo buccinador para entender la movilidad facial. En todos los casos, encontraron que la inserción principal de la banda muscular profunda del músculo cigomático mayor fue en el margen anterior del buccinador y su fascia. Esta relación anatómica proporciona la tracción sincrónica de la región anterior del buccinador con la correspondiente mucosa oral hacia afuera y hacia arriba para crear la sonrisa (Shim et al.).

En relación a la inervación de los músculos que conforman el modiolo del ángulo oral, específicamente el buccinador, clásicamente se lo considera inervado por ra- mos bucales del nervio facial (Pró, 2012), sin embargo, es necesario destacar lo establecido por Giacomotti et al. Estos autores identifican una doble inervación del buccinador: mitad superior, inervada por ramos bucales del nervio facial, al igual que el músculo levantador del ángulo oral; y su mitad inferior, por la rama marginal mandibular del nervio facial, al igual que el depresor de ángulo oral. De esta manera, se puede precisar la funcionalidad del modiolo del ángulo oral en los movimientos de la comisura labial. La lesión del ramo maginal mandibular del nervio facial, debilitaría el mecanismo de contención labial, con flacidez de la mejilla y a aparición de sialorrea (Giacomotti et al.). La elevación de la comisura no se vería afectada, ya que se mantendría intacta la inervación del músculos intervinientes en la oclusión-proyección labial [el músculo cigomático mayor y el complejo muscular "levantador-depresor" del ángulo oral (Giacomotti et al.), constituído por los músculos levantador del ángulo oral y depresor del ángulo oral, y la porción superior del músculo buccinador] (Ewart et al.; Giacomotti et al.). Así, el paciente, a pesar de la lesión, podrá silbar o besar (Giacomotti et al.).

Continuando con esta idea funcional, para Choi et al. el modiolo del ángulo oral está directamente involucrado en todas las fuerzas que actúan sobre la comisura labial. Por lo tanto, la hiperactividad del depresor del ángulo oral puede llevar hacia inferior la posición modiolar, dando lugar a la caída de la comisura labial, lo que puede manifestarse en una expresión deprimida y sombría. Según Choi et al. la aplicación de toxina botulínica tipo A al depresor del ángulo oral, provocando su inactivación, podría llevar a la elevación de la comisura labial por los músculos antagonistas, como ser el levantador del ángulo oral y el músculo cigomático mayor, que participan en la formación del modiolo del ángulo oral.

Clínicamente, Tong et al. demostraron que durante el desarrollo de colgajos, si se mantiene el modiolo del ángulo oral, los fascículos musculares que lo conforman, al presentar varias direcciones de orientación, logran simular la acción del esfínter oral en el seguimiento a largo plazo. Consideran importante que el músculo buccinador se permanezca lo menos afectado posible, con preservación de la inervación con un colgajo de avance de la mejilla diseñado adecuadamente. Tong et al. consideran que aunque se reseca la mayor parte de la porción labial inferior del músculo orbicular oral, la conservación de los músculos buccinador, depresor del ángulo oral y cigomático mayor en bloque, es decir, la conservación del modiolo del ángulo oral, se asegurará la preservación de la contracción muscular compensatoria y el movimiento en todas las direcciones. Aunque el músculo depresor del ángulo oral y ocasionalmente, el músculo cigomático mayor se seccionan durante 
el avance, es fundamental la conservación de su fijación en el modiolo del ángulo oral, lo cual permite alguna función después de que el extremo proximal de la unidad muscular se haya fibrosado. A su vez, Shim et al. indicaron que es fundamental que el nuevo músculo o tendón que se utilicen en la corrección de las lesiones de la región, se unan a los músculos periorales, por debajo del músculo levantador del ángulo oral, asegurando la sutura con el buccinador, de esta manera, se logrará recrear la apariencia natural del paciente, y la preservación de la función facial.

Kwak et al. (2006) estudiaron la relación de la arteria facial con el músculo cigomático mayor. En este sentido, identificaron que durante los procedimientos de cirugía plástica de la cara, como el estiramiento facial y el colgajo musculomucoso de la arteria facial, la relación entre la arteria facial y el músculo cigomático mayor es crítica. Las variaciones en el trayecto de la arteria facial deben considerarse para el diseño del colgajo y es importante no destruir su continuidad.

Por su parte, Hur describió el hallazgo del músculo incisivus labii superioris (ILS) en todas las muestras estudiadas (52 en total). De esta manera, describió su origen oblicuo y lineal en la fosa incisiva del paladar óseo hasta un punto medial al origen del músculo levantador del ángulo oral. Las fibras musculares del ILS se arquean y cubren las glándulas labiales en el margen superior del músculo orbicular oral. De esta manera, identificó que el ILS discurre lateralmente a lo largo de la parte anterior del pliegue mucolabial superior; sus fibras a su vez se mezclan con varios músculos en el modiolo del ángulo oral, interviniendo por lo tanto en su conformación. Y además estableció que el ILS está ubicado entre el músculo orbicular oral y el levantador del ángulo oral en una disposición continua.

\section{CONCLUSIONES}

El conocimiento preciso de la disposición topográfica del modiolo del ángulo oral es esencial para la planificación quirúrgica y para el desarrollo de procedimientos como la mioplastia o el trasplante de músculo libre (Shim et al.). En el rejuvenecimiento perioral, además es importante comprender el movimiento de la comisura labial y el equilibrio de fuerzas ejercidas por los músculos que constituyen el modiolo del ángulo oral (Ewart et al.; Sullivan et al., 2010). Además, la comprensión del conocimiento anatómico de la expresión facial es importante en el tratamiento de la parálisis facial (Shim et al.). Un modiolo del ángulo oral trófico y grande, puede mantener el cuerpo adiposo en su posición original y evitar que sobresalga (Sullivan et al., 2010). De esta manera, en caso de requerir la intervención quirúrgica del modiolo del ángulo oral, su ubicación en una posición más elevada, provocaría la elevación de la comisura, mejorando la simetría facial tanto en reposo como en movimiento (Ewart et al.).

El pliegue nasolabial también está asociado con el envejecimiento facial y está íntimamente conectado con el modiolo del ángulo oral; un pliegue nasolabial convexo largo aparece en algunos casos con un modulo débil y trófico, mientras que un pliegue nasolabial cóncavo corto aparece en casos con un modiolo del ángulo oral grande y trófico (Kim et al.).

A partir de los estudios analizados, se considera la siguiente disposición en planos de los músculos que conforman el modiolo del ángulo oral: plano superficial, involucra al complejo "levantador-depresor" del ángulo oral, conformado por el músculo levantador del ángulo oral y el músculo depresor del ángulo oral; plano intermedio, representado por el músculo cigomático mayor; plano profundo, constituido por las dos mitades del músculo buccinador.

OTTONE, N. E. Preliminary communication about the morphological arrangement, functionalitand clinical implications of modiolus of angulus oris (Modiolus anguli oris). Int. J. Morphol. 38(6):1837-1841, 2020.

SUMMARY: The anatomical arrangement of the facial muscles is of considerable complexity and requires a detailed study to achieve its precise recognition. The understanding of the morphological disposition and the functionality of the angle of the mouth (Modiolus angulis oris) is of great relevance when analyzing the muscular constitution of the labial commissure, and to understand clinically the injuries of the angulus oris, what that will allow an adequate therapeutic approach, crucial for the preservation and reconstitution of facial harmony.

KEY WORDS: Modiolus; Modiolus angulis oris; Morphology; Clinical Anatomy.

\section{REFERENCIAS BIBLIOGRÁFICAS}

Al-Hoqail, R. A. \& Abdel Meguid, E. M. An anatomical and analytical study of the modiolus: enlightening its relevance to plastic surgery. Aesthetic Plast. Surg., 33(2):147-52, 2009.

Choi, Y. J.; Kim, J. S.; Gil, Y. C.; Phetudom, T.; Kim, H. J.; Tansatit, T. $\& \mathrm{Hu}, \mathrm{K}$. S. Anatomical considerations regarding the location and boundary of the depressor anguli oris muscle with reference to botulinum toxin injection. Plast. Reconstr. Surg., 134(5):917-21, 2014.

D'Andrea, E. \& Barbaix, E. Anatomic research on the perioral muscles, functional matrix of the maxillary and mandibular bones. Surg. Radiol. Anat., 28(3):261-6, 2006. 
Ewart, C. J.; Jaworski, N. B.; Rekito, A. J. \& Gamboa, M. G. Levator anguli oris: a cadaver study implicating its role in perioral rejuvenation. Ann. Plast. Surg., 54(3):260-3, 2005.

Freilinger, G.; Gruber, H.; Happak W. \& Pechmann, U. Surgical anatomy of the mimic muscle system and the facial nerve: importance for reconstructive and aesthetic surgery. Plast. Reconstr. Surg. 80(5):686-90, 1987.

Giacomotti, J. D.; Bertone, V. H.; Conesa, H. A.; Ouviña, J. M.; Seiler, J. I.; Ottone, N. E.; Domínguez, M. L. \& Arrotea, A. Nuevos conceptos sobre el sistema muscular peribucal. Cir. Plast. Iberolatinoam., 35(2):101-6, 2009.

Hur, M. S. Anatomical features of the incisivus labii superioris muscle and its relationships with the upper mucolabial fold, labial glands, and modiolar area. Sci. Rep., 8(1):12879, 2018.

Kim, H. S.; Pae, C.; Bae, J. H.; Hu, K. S.; Chang, B. M.; Tansatit, T. \& Kim, H. J. An anatomical study of the risorius in Asians and its insertion at the modiolus. Surg. Radiol. Anat., 37(2):147-51, 2015.

Kwak, H. H.; Hu, K. S.; Youn, K. H.; Jin, G. C.; Shim, K. S.; Fontaine, C. \& Kim, H. J. Topographic relationship between the muscle bands of the zygomaticus major muscle and the facial artery. Surg. Radiol. Anat., 28(5):477-80, 2006.

Lightoller, G. H. Facial muscles: the modiolus and muscles surrounding the rima oris with some remarks about the panniculus adiposus. $J$. Anat., 60(Pt. 1):1-85, 1925.

Pró, E. A. Anatomía Clínica. Buenos Aires, Médica Panamericana, 2012. pp.244, 248.

Rubin, L. R. The anatomy of a smile: its importance in the treatment of facial paralysis. Plast. Reconstr. Surg., 53(4):384-7, 1974.

Shim, K. S.; Hu, K. S.; Kwak, H. H.; Youn, K. H.; Koh, K.; Fontaine, C. \& Kim, H. J. An anatomical study of the insertion of the zygomaticus major muscle in humans focused on the muscle arrangement at the corner of the mouth. Plast. Reconstr. Surg., 121(2):466-73, 2008.

Sun, M.; Chen, G. C.; Wang, Y. Q.; Song, T.; Li, H. D.; Wu, D. \& Yin, N. B. Anatomical characterization and three-dimensional modeling of the muscles at the corner of the mouth: an iodine staining technique based on micro-computed tomography. Plast. Reconstr. Surg., 142(3):782-5, 2018.

Tong, C. C. L.; Vandegriend, Z. P.; Lee, Y. H. \& Lawson, W. Anatomical basis for lip reconstruction: the role of the modiolus. Ann. Plast. Surg., 82(5):565-569, 2019.

Williams, P. L. Anatomía de Gray. Bases Anatómicas de la Medicina y la Cirugía. $38^{\text {a }}$ ed. Madrid, Churchill Livingstone, 1998.

Yu, S. K.; Lee, M. H.; Kim, H. S.; Park, J. T.; Kim, H. J. \& Kim, H. J. Histomorphologic approach for the modiolus with reference to reconstructive and aesthetic surgery. J. Craniofac. Surg., 24(4):14147, 2013.

\author{
Dirección para correspondencia: \\ Dr. Nicolás E. Ottone (M.D., Ph.D.) \\ Profesor Asociado \\ Laboratorio de Plastinación y Técnicas Anatómicas \\ Facultad de Odontología \\ CEMYQ \\ Universidad de La Frontera \\ Temuco \\ CHILE
}

E-mail: nicolas.ottone@ufrontera.cl

Website: www.plastinacion.com

Recibido: 05-05-2020

Aceptado:18-06-2020 